\title{
Involvement of both prefrontal and inferior parietal cortex \\ in dual-task performance
}

\author{
Fabienne Collette $^{\mathrm{a}, \mathrm{b}}$, Laurence 01ivier ${ }^{\mathrm{b}, \mathrm{c}}$, Martial Van der Linden ${ }^{\mathrm{a}, \mathrm{d}}$, Steven Laureys ${ }^{\mathrm{b}}$, Guy Delfiore ${ }^{\mathrm{b}}$, André \\ Luxen $^{\mathrm{b}}$, Eric Salmon ${ }^{\mathrm{b}, \mathrm{c}}$ \\ ${ }^{a}$ Department of Neuropsychology, University of Liège, Belgium \\ ${ }^{\mathrm{b}}$ Cyclotron Research Center, University of Liège, Belgium \\ ${ }^{c}$ Department of Neurology, University of Liège, Belgium \\ ${ }^{\mathrm{d}}$ Cognitive Psychopathology Unit, University of Geneva, Switzerland
}

\begin{abstract}
This PET study explored the neural substrate of both dual-task management and integration task using single tasks that are known not to evoke any prefrontal activation. The paradigm included two simple (visual and auditory) discrimination tasks, a dual task and an integration task (requiring simultaneous visual and auditory discrimination), and baseline tasks (passive viewing and hearing). Data were analyzed using SPM99. As predicted, the comparison of each single task to the baseline task showed no activity in prefrontal areas. The comparison of the dual task to the single tasks demonstrated left-sided foci of activity in the frontal gyrus (BA 9/46, BA 10/47 and BA 6), inferior parietal gyrus (BA 40), and cerebellum. By reference to previous neuroimaging studies, BA 9/46 was associated with the coordinated manipulation of simultaneously presented information, BA 10/47 with selection processes, BA 6 with articulatory rehearsal, and BA 40 with attentional shifting. Globally similar regions were found for the integration task, except that the inferior parietal gyrus was not recruited. These results confirm the hypothesis that the left prefrontal cortex is implicated in dual-task performance. Moreover, the involvement of a parietal area in the dual task is in keeping with the hypothesis that a parieto-frontal network sustains executive functioning.
\end{abstract}

Theme: Neural basis of behavior

Topic: Cognition

Keywords: Positron emission tomography; Dual task; Executive functions; Prefrontal cortex

\section{INTRODUCTION}

The ability to coordinate concurrent cognitive processes is a crucial executive function [4]. This function has commonly been investigated using dual-task paradigms in which the subjects' performance is compared during the successive and simultaneous execution of two separate tasks [3,9]. Such paradigms have demonstrated that the simultaneous performance of two tasks leads to increased response times and more errors. A failure to simultaneously perform two tasks is a characteristic feature of the dysexecutive syndrome exhibited by some patients with frontal lesions [5,19]. Although dual-task management is considered one of the main functions of the central executive of working memory, this dual processing is not limited to tasks requiring information storage in working memory, but also intervenes in perceptual tasks requiring no memory load (e.g., [12,41]). Classically, a distinction was made between dual tasks-which require subjects to combine two tasks that are unrelated to each other-and coordination or integration tasks-which require the integration of information coming from different sources (e.g., [60]; see, however, [24]).

Several controversies exist about the exact mechanisms involved in dual-task performance. Following the response-selection bottleneck theory, if a person is engaged in selecting the response to a stimulus for one task, then selecting another response to a different stimulus for a second task cannot proceed until the first response- 
selection process has finished (e.g., [43]). In contrast, models of adaptive executive control consider that declarative set of rules for performing individuals tasks are acquired through practice and are converted to procedural knowledge. Consequently, dual-task interference can stem from an incomplete conversion of declarative to procedural knowledge or from executive control that give priority on one of the processes according to task instructions [39]. In agreement with the first hypothesis, Garavan [28] showed that performing a dual-count task results in a sizeable time cost that persists even after intensive practice, which indicates that people do not have simultaneous access to all items currently in working memory. On the contrary, Schumacher et al. [51] demonstrated that after practice, perfect time sharing in dual-task performance was observed and that interference between tasks can be modulated by instructions about differential tasks priorities, which is consistent with the proposal of Meyer and Kieras [39]. However, the timing of the dual process is different in these two studies, which could explain these apparently discrepant results. In the Schumacher et al. study, subjects have to perform both tasks simultaneously while there is no overlap between the two kinds of items to be processed in Garavan's study.

With regard to functional neuroimaging, two potential mechanisms have been proposed to explain the deterioration in performance on dual-task paradigms relative to separate performance of each task: (1) dual-task paradigms may require additional cognitive operations and activation of specific brain regions in addition to those activated by the performance of single tasks alone; (2) the two tasks may interfere (and thus increase reaction times) if they recruit the same population of neurons at the same time or if they activate distinct neural populations (within the same brain region) that mutually inhibit each other when recruited simultaneously [34]. The first study to explore cerebral areas associated to dual-task performance was that of D'Esposito et al. [20]. These authors compared cerebral activity when two non-working-memory tasks were performed in isolation or simultaneously. Tasks used were a semantic judgment task and a spatial-rotation task, which were considered as activating predominantly posterior brain regions ([22,30]; see, however, [33]). The simultaneous execution of both tasks involved significant increases of activity bilaterally in the dorsolateral prefrontal cortex (BA 9 and 46) and the anterior cingulate region. These data support the hypothesis that the dorsolateral prefrontal cortex is involved in the allocation and coordination of attentional resources. More recently, Herath et al. [31] also demonstrated that performance of dual-reaction-time tasks activates cortical regions in addition to those activated by the performance of component single tasks (namely, visual and somatosensory detection tasks). These brain activations were located bilaterally in the superior frontal cortex, but also in the intraparietal sulcus and the supramarginal gyrus.

However, other studies did not demonstrate additional prefrontal activity during dual-task performance. Klingberg [34] showed that the simultaneous performance of two (visual and auditory) working-memory tasks did not require supplementary cerebral areas in comparison to the performance of the single tasks. These data were confirmed in other studies using working-memory tasks (the reading span test [10] and the operation span test [54]) as well as in a study using tasks that did not require storage of the information to be simultaneously processed [1]. These results seem to indicate that no precise cortical area is associated with any specific cognitive process for dual-task performance and that the simultaneous execution of two tasks depends mainly upon greater activity of $[1,34]$ or interaction between $[10,54]$ the cerebral areas already activated for the single tasks.

Finally, Just et al. [33] have demonstrated that cerebral activity during the simultaneous realization of two tasks was substantially less than the sum of the activation when each task was performed alone. This was observed not only in primary and secondary sensory areas but also in association areas (primarily parietal and temporal cortex). These results indicate that the dual-task condition induces some mutual constraints among cortical areas. The authors interpreted these data as suggesting the existence of biological mechanisms that place an upper limit on the amount of cortical tissue which can be activated at any given time, thereby resulting in a limit on how much attention is available to distribute over concurrent tasks.

As indicated previously, some authors have made a distinction between dual tasks (simultaneous processing of two kinds of information) and integration tasks (combining information coming from different sources). Unlike dual-task management processes, few studies have explored the neural substrates of the integration process. Prabhakaran et al. [46] used fMRI to identify the brain regions preferentially involved in maintaining integrated versus unintegrated spatial and verbal information in working memory. Their results indicate that the right middle and superior frontal gyri (BA 9, 10, 46) were more involved in maintaining integrated information, while the maintenance of unintegrated information required greater involvement by the posterior cerebral areas. In another study, Mitchell et al. [40] confirmed the involvement of the right medial prefrontal cortex (BA 10) and also demonstrated greater activation in the left anterior hippocampus when participants had to remember objects together with their location than on trials in which participants were told to remember either object or location 
information but not both. Moreover, both the anterior cingulate (BA 24/32) and left precentral gyrus/ premotor cortex (BA 6) were also more activated in the integration condition.

\section{AIMS}

Previous studies exploring the neural substrates of dual-task performance have provided contradictory results. The presence or absence of prefrontal activity in a dual task cannot be explained by the relative difficulty of the various dual-task paradigms used. Indeed, in all the studies mentioned above [1,10,20,31,34,54], subjects experienced a decrease in performance from the single tasks to the dual task. The only study that clearly demonstrated that frontal activation was related to behavioral interference costs was that of Smith et al. [54], in which only subjects with the lower dual-task performance recruited the left dorsolateral prefrontal cortex. One possible explanation to these discrepancies may be the use of single tasks which already involved executive functioning. Indeed, activity in the frontal areas was already present during single-task performance in most of these studies. Consequently, we explored dual-task management by using simple verbal and auditory discrimination tasks that are not supposed to elicit prefrontal activation when performed in isolation. ${ }^{1}$ In that way, we should formally determine whether dual-task management involves supplementary activity at the level of the prefrontal cortex or greater activity only in the posterior cerebral areas already activated by the single tasks. Moreover, we were also interested in determining whether dual-task management and integration processes depend on similar or different cerebral areas, since the neural substrates of these two kinds of paradigms have hitherto been explored in separate studies. Dual and integration tasks were built-up in order to minimize the working-memory storage requirements. Indeed, storage in working memory was found to be associated with recruitment of prefrontal areas (e.g., [8]). Moreover, the neural substrates of the integration process were explored earlier using working-memory paradigms and we were interested in determining whether the results obtained would be similar with perceptual tasks.

\section{MATERIAL AND METHODS}

\subsection{Subjects}

Thirteen right-handed volunteers ( 7 males, age ranging from 21 to 25 years) gave written informed consent to take part in this study, which was approved by the University of Liège Ethics Committee. None of the subjects had a history of psychiatric or neurological illness. None were on medication at the time of testing.

\subsection{Positron emission tomography scanning and data processing}

PET data were acquired on a Siemens CTI 951 R 16/31 scanner in 3D mode. The subject's head was stabilized by a thermoplastic face mask secured to the head holder (Truscan imaging, MA), and a venous catheter was secured in a left antebrachial vein. First, a 20-min transmission scan was acquired for attenuation correction using three rotating sources of $68 \mathrm{Ge}$. Then, regional cerebral blood flow, taken as a marker of local neuronal activity, was estimated during 12 emission scans. Each scan consisted of two frames: a 30-s background frame and a 90-s acquisition frame. The slow intravenous water $\left(\mathrm{H}_{2}{ }^{15} \mathrm{O}\right)$ infusion began $10 \mathrm{~s}$ before the second frame. Six mCi $(222 \mathrm{MBq})$ were injected for each scan, in $5 \mathrm{cc}$ saline, over a period of $20 \mathrm{~s}$. The infusion was totally automated in order not to disturb the subject during the scanning period. Data were reconstructed using a Hanning filter (cutoff frequency: 0.5 cycle/ pixel) and corrected for attenuation and background activity. The cognitive tasks were randomly distributed among subjects, with the exception that no cognitive task was administered twice in succession and that the two executive tasks (dual task and integration task) were not performed successively.

\subsection{Cognitive tasks}

The experimental design was composed of five conditions: baseline, auditory and visual discrimination tasks, dual task (simultaneous execution of the two discrimination tasks), and integration task. For each task, a similar number of auditory and visual stimuli were presented. Two versions of the baseline and sensory discrimination tasks and three versions of the integration and dual tasks were created and administered to subjects (for a total of 12 scans per session).

\footnotetext{
${ }^{1}$ A few other studies have explored the neural substrates of dual-task management using simpler tasks [37,55]. However, these studies also showed activity in frontal areas during the performance of single tasks, which complicated the interpretation of the supplementary prefrontal activity found in dual-task management.
} 
Visual stimuli consisted of crosses presented in the bottom, middle, or top of a computer screen. Auditory stimuli were low $(220 \mathrm{~Hz})$, medium $(440 \mathrm{~Hz})$, or high $(880 \mathrm{~Hz})$ tones presented through earphones. In each task, a cross and a tone were simultaneously presented. In the discrimination tasks, subjects had to process only one kind of stimulus (that was indicated before starting the task). In the dual and integration tasks, both kinds of stimuli had to be processed. Each task lasted $120 \mathrm{~s}$ and was composed of 24 trials. The stimuli were presented via E-prime software [49]. Data were collected by a response box which was connected to the computer and which recorded response time and number of correct responses.

Fig. 1. Illustration of the time course of the experimental conditions.

\section{Target items Cues}

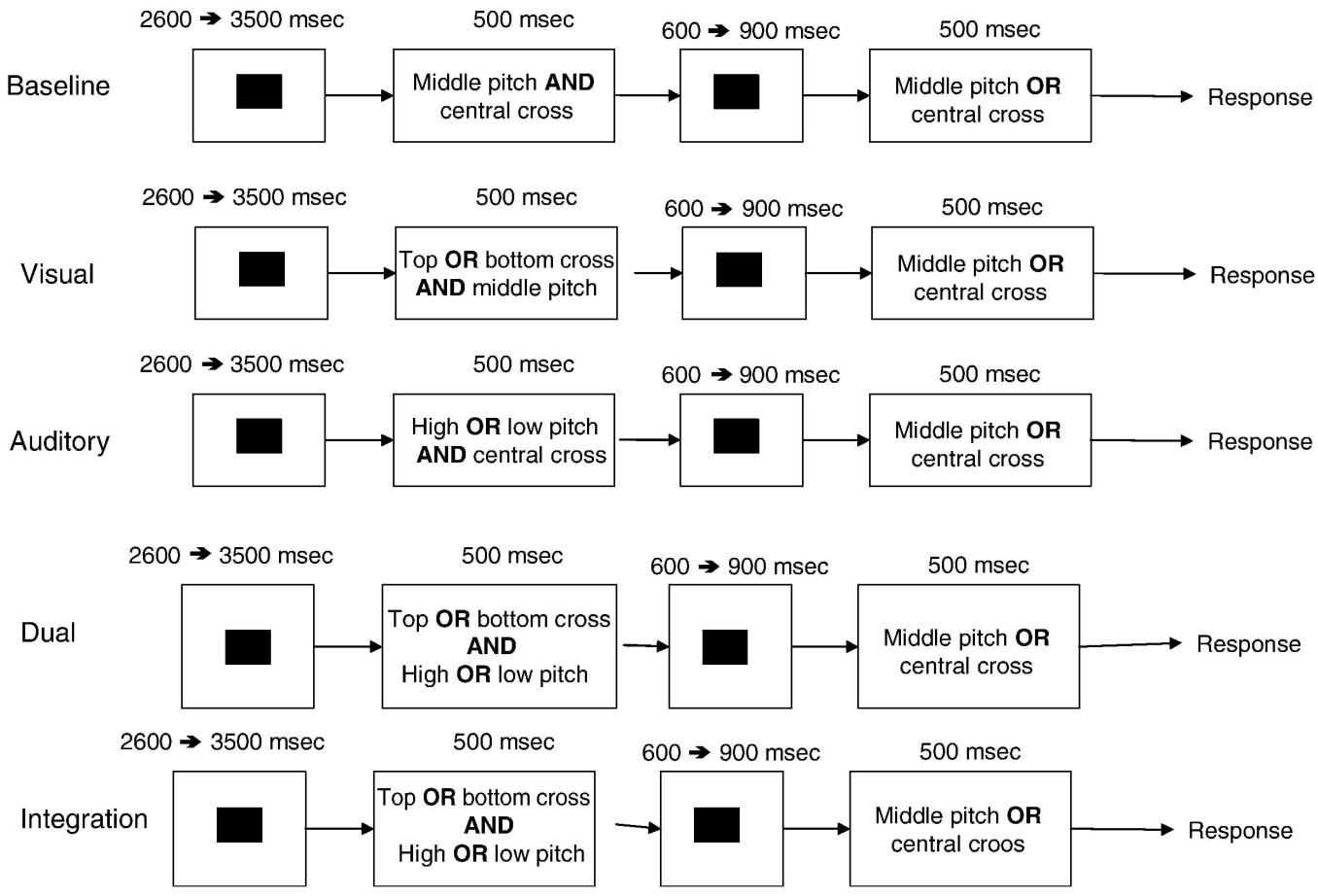

The procedure used in the different tasks was the following (see Fig. 1). First, a fixation point (black square) was presented for a time interval ranging from 2600 to $3500 \mathrm{~ms}$ (in increments of $300 \mathrm{~ms}$ ). Next, the target item was presented; it consisted of the simultaneous presentation of a cross and a tone for $500 \mathrm{~ms}$. The fixation point was again presented for 600,750 , or $900 \mathrm{~ms}$, followed by a cue signal. The cue signal consisted of a 500-ms presentation of a medium-pitched tone or a cross in the center of the screen, prompting the subject to produce the response. The response consisted of pressing one of two response keys on the basis of the sensory characteristic of the target stimuli (in other words, high- or low-pitched tone; cross in the top or bottom part of the screen).

It should be noted that, although two target stimuli were presented in the dual-task condition, subjects subsequently responded to only one of them (indicated by the cue signal), so that the number of motor responses would be similar in the five conditions. Moreover, in order to present a similar number of auditory and visual stimuli in the various tasks, the cue signal was presented in all conditions and neutral visual and auditory stimuli (namely, medium-pitched tone and centrally presented cross) were presented in the single tasks in association with the target stimuli. Two criticisms could nevertheless be made of this experimental design. First, the simultaneous presentation of visual and auditory information in the single discrimination tasks is likely to induce interference, and thus executive demand, in our simple tasks. However, the same non-relevant (or neutral) stimulus (middle-pitch tone or centrally presented cross) was presented throughout all the task and it was shown that there are very few interference costs following the successive presentation of the same interfering item [38]. 
Second, there was a short delay between the presentation of the target item(s) and the production of the response, involving the short-term storage of one piece of information in the discrimination and integration tasks and two pieces of information in the dual task. It was previously demonstrated that such a low memory load only involves the ventrocaudal frontal regions [48]. Consequently, the introduction of the delay for maintenance of minimal information should not result in activity in the prefrontal regions, and any changes in activity observed should be related to dual-task management.

\subsubsection{Baseline task}

In this condition, target items consisted of the simultaneous presentation of centrally presented crosses and medium-pitched tones. After a delay, the cue signal was presented. In half of the trials, the cue was the centrally presented cross, and in the other half, it was the mid-pitch tone. The two kinds of cues were randomly presented. The subject's response involved pressing one response key following the cue.

\subsubsection{Visual discrimination task}

Target items comprised a medium-pitch tone associated with the presentation of a cross in the top or bottom portion of the screen. Crosses were equally and randomly presented in both parts of the screen. Again, a cue signal was presented after a delay and, as in the baseline task, this cue consisted of the centrally presented cross in half of the trials and the medium-pitched tone in the other half. Following presentation of the cue, subjects had to press one of the two response keys to indicate whether the cross was presented in the top or bottom part of the screen.

\subsubsection{Auditory discrimination task}

In this condition, target items were composed of a centrally presented cross associated with a high- or low-pitch tone. The two kinds of tones were equally and randomly presented. After a variable delay, the same cues as in the baseline task and the auditory discrimination task were presented and subjects pressed a key to indicate whether the tone was low- or high-pitched.

\subsubsection{Dual task}

This condition consisted of the simultaneous processing of auditory and visual information. Target items were high-and low-pitch tones associated with crosses presented in the lower or upper part of the screen. After a delay, the cue was presented. When the cue was the medium-pitch tone, subjects had to press a key to indicate whether the target tone was high- or low-pitch, while when the cue was a centrally presented cross, subjects had to indicate whether the target cross was presented in the bottom or top of the screen. This procedure required subjects to simultaneously process and temporarily maintain both visual and auditory information. On the basis of task analysis, the dual-task condition, in comparison to the single discrimination tasks, specifically requires the simultaneous processing of two different kinds of information, as well as the storage of these pieces of information in working memory. Finally, upon presentation of the cue, subjects have to select the sensory information relevant to produce a response, and consequently inhibit their response to the other sensory representation. Another cognitive component that could be involved in the task is switching between the two representations stored in working memory. Indeed, some data [28] seem to indicate that we are limited to paying attention to just one representation in working memory, which consequently necessitates constant switches between the different objects stored in working memory.

\subsubsection{Integration task}

As in the dual-task condition, target items were high- and low-pitch tones associated with crosses presented in the lower or upper part of the screen. However, this time, subjects did not have to process auditory and visual information in parallel during the delay; rather, they had to integrate the two kinds of information to decide whether the two stimuli were congruent or not. We defined a congruent pair as composed of a cross presented in the upper portion of the screen and a high-pitch tone (both items are "at the top") or a cross presented in the lower part of the screen paired with a low-pitch tone (both items are "at the bottom"). Incongruent pairs corresponded to the other combinations of visual and auditory items. After a variable delay, a cue was presented (medium-pitch tone or centrally presented cross) and subjects had to press a key to indicate whether the previously presented pair of items was congruent or not. The integration requires, in comparison to the single tasks, the simultaneous processing of two kinds of information. This process was also involved in the dual task. Processes specific to the integration task are the detection of a match between visual and auditory information 
and the storage of the response until the presentation of the probe. The main difference between this task and the tasks used by Prabakharan et al. [46] and Mitchell et al. [40] is that these studies require subjects to bind a series of spatial and verbal information and keep this information in working memory while the integration process, in the sense used here, involves perceptually matching two pieces of information and briefly keeping the result of this match in working memory.

Subjects were given one short training session five or six days before the PET session. Three minutes before each acquisition, the instructions were rehearsed. After the scanning session, post hoc questioning of the subjects indicated that they had perfectly complied with the task instructions. Each of the tasks was performed two or three times (with a total of 12 scans) during the session and was counterbalanced between subjects to control for practice and weariness effects.

\subsection{Data analysis}

Data were analyzed using statistical parametric mapping (SPM99; Welcome Department of Cognitive Neurology, Institute of Neurology, London, UK; http://www.fil.ion.ucl. ac.uk/spm) implemented in MATLAB. For each subject, all scans were realigned together, then normalized to a standard PET template using the same transformations. Finally, PET images were smoothed using a Gaussian kernel of $16 \mathrm{~mm}$ full width at half maximum to accommodate intersubject differences in gyral and functional anatomy and to suppress highfrequency noise in the images. Such transformations of the data allow for voxel-by-voxel averaging of data across subjects and for direct cross-reference to the anatomical features in the standard stereotactic atlas [56]. Coordinates of foci of activation obtained in the SPM space were finally transformed to correspond to coordinates in the Talairach space [56].

Differences in global activity within and between subjects were removed by analysis of covariance on a voxelby-voxel basis with global count as covariate and regional activity across subjects for each task as treatment [25]. The across-task comparisons were first performed by averaging between paired measurements (fixed effect model). For each voxel in stereotactic space, the analysis of covariance (ANCOVA) generated a conditionspecific adjusted mean regional cerebral blood flow (rCBF) value (normalized to $50 \mathrm{ml} / 100 \mathrm{ml} / \mathrm{min}$ ) and an associated adjusted error variance. The ANCOVA allowed the comparison of the means across conditions on a voxel-by-voxel basis using the $t$ statistic. The resulting sets of $t$ values constituted statistical parametric maps $[\operatorname{SPM}(t)][26]$. The $\operatorname{SPM}(t)$ were transformed to the unit normal distribution [SPM(Z)].

The design of our study was a cognitive subtraction paradigm. In this design, the visual and auditory discrimination tasks were compared to each other as well as to the baseline in order to determine the cerebral areas associated with auditory and visual sensory processes. The dual and integration tasks were compared in two separate analyses to the two sensory discrimination tasks in order to determine which cerebral areas were associated with these executive processes: [dual — (auditory discrimination + visual discrimination)] and [integration - (auditory discrimination + visual discrimination)]. These two executive tasks were also compared to each other to determine which cerebral areas were specifically associated with each of them. The comparisons including dual and integration tasks were also performed using a random effect model [32], in order to determine exactly which cerebral areas are likely to be activated in the population and are not due to idiosyncratic strategies of some subjects. The random effect model is based on the approach of mean summary statistics on repeated measures [27]. This type of analysis is usually used in neuroimaging studies to accommodate both interindividual and intraindividual variability of PET data and requires that all members of the population show this effect so that its expectation is greater than under the null hypothesis.

We used an SPM with a threshold of $P<0.05$ (voxel level, with Bonferonni correction for multiple comparisons). The corrected $t$ threshold was set at 3.16 for fixed effects and 3.93 for random effects. The SPM threshold was $P<0.001$ (uncorrected for multiple comparisons), when looking for activation in a region predicted by previous studies. In the case of the integration tasks, a small volume correction [59] was computed on a 10-mm sphere around the average coordinates published for the corresponding relevant a priori location (for the hippocampus: $x=-33, y=-14, \mathrm{z}=-11$ [40]; for the medial frontal cortex $x=6, y=54, \mathrm{z}=13$ [40]). Statistical inferences were obtained at the voxel level $P<0.05$, over the volume of interest. 


\section{RESULTS}

\subsection{Cognitive performance}

The number of correct responses and mean response times were collected for the different tasks (see Table 1). Repeated measure analyses of variance showed that response time and number of correct answers differed significantly for the five conditions [respectively, $F(4,48)=77.63, P<0.00001$ and $F(4,48)=3.08, P<0.05$ ] Planned comparisons demonstrated that response times on the baseline tasks were faster than on the two single tasks [auditory: $F(1,12)=7.48, P<0.05$; visual: $F(1,12)=3.71, P=0.08$ ], and that dual and integration tasks were also performed more slowly than the two single tasks [auditory vs. dual: $F(1,12)=82.16, P<0.0001$; visual vs. dual: $F(1,12)=82.16, P<0.0001$; auditory vs. integration: $F(1,12)=5.21, P<0.05$; visual vs. integration: $F(1,12)=7.29, P<0.05]$. Finally, response times were slower in the dual task than in the integration condition $[F(1,12)=89.07, P<0.0001]$. With regard to correct responses, the only significant difference was between the integration task and the two single tasks [auditory: $F(1,12)=9.78, P<0.01$; visual: $F(1,12)=4.45, P=0.05]$.

\subsection{Imaging data}

\subsubsection{Single tasks minus baseline}

In a first analysis, changes in $\mathrm{rCBF}$ during the visual and auditory discrimination tasks were compared to the baseline. This was done to be sure that no prefrontal areas were already activated during the performance of the single tasks. At the threshold of $P<0.001$ (uncorrected for multiple comparisons, to detect even slight prefrontal recruitment), the only anterior area found was the caudate nucleus (visual task: coordinates $12,22,0, \mathrm{Z}$ score -3.17 ; auditory task: coordinates $14,20,2, \mathrm{Z}$ score -3.79 ). When the statistical threshold was further lowered at $P<0.05$ (uncorrected), no supplementary prefrontal activity was found in the visual and auditory tasks.

It should be noted that no significant foci of activity were observed for the two discrimination tasks at the corrected $P<0.05$ level when compared to the baseline. However, at $P<0.001$ uncorrected, foci of activity were observed bilaterally in the superior parietal gyrus for the visual task as well as in the right superior/middle temporal gyrus (BA 21/22) for the auditory task and in the right middle and superior occipital (BA 19) (see Table 2). Previous work exploring visual and auditory information processing demonstrated activity in these areas $[6,37,42]$,

\subsubsection{Comparison of the two single discrimination tasks}

These analyses $(P<0.001$, uncorrected $)$ were performed to determine which cerebral areas were relatively more involved in auditory or visual discrimination processes (see Fig. 2.1). Increases in cerebral activity for the visual task in comparison to the auditory task were found in the right angular gyrus (BA 39), the right middle occipital gyrus (BA 19), the right superior parietal gyrus (BA 7), and the right fusiform gyrus (BA 20) (see Table 3). On the other hand, the auditory discrimination task was more associated with the right superior temporal gyrus (BA 22) and the inferior occipital gyrus bilaterally (BA 18) (see Table 4). Moreover, plots of activity in these regions were determined for the different conditions (see Fig. 3). These plots indicated that cerebral activity in these regions did not increase during the dual-task and integration conditions but rather is decreased in all regions at the exception of BA 7.

Table 1. Subjects' cognitive performance in the five conditions

\begin{tabular}{llllll}
\hline & Baseline & Auditory & Visual & Dual & Integration \\
\hline Response times & $236 \pm 45$ & $266 \pm 63$ & $262 \pm 78$ & $748 \pm 231$ & $309 \pm 110$ \\
Correct responses & $23.5 \pm 1.2$ & $23.8 \pm 0.6$ & $23.4 \pm 0.8$ & $22.5 \pm 1.4$ & $22.5 \pm 2.2$ \\
\hline
\end{tabular}

Response times in milliseconds (mean \pm standard deviation) and number of correct responses (maximum $=24$; mean \pm standard deviation) in the five conditions. Auditory = single auditory discrimination task; Visual = single visual discrimination task; Dual = dual-task management (simultaneous processing of visual and auditory information); Integration = integration task (integration of simultaneously presented visual and auditory information). 
Table 2. Regions with significant $r C B F$ changes in discrimination tasks versus baseline

Brain areas $\quad \frac{\text { Stereotactic coordinates }}{x}$ Z score

\begin{tabular}{lcccc}
\hline (A) Visual discrimination $v$ s. baseline & & & & \\
Voxel $P$ value $<0.001$, uncorrected for multiple comparisons & & \\
R middle and superior occipital & 38 & -69 & 22 & 3.31 \\
$\quad$ gyrus (BA 19) & 42 & -70 & 29 & 3.68 \\
R superior parietal gyrus (BA 7) & 16 & -56 & 45 & 3.47 \\
& 18 & -58 & 53 & 3.60 \\
L superior parietal gyrus (BA 7) & -20 & -61 & 55 & 3.36
\end{tabular}

(B) Auditory discrimination vs. baseline

Voxel $P$ value $<0.001$, uncorrected for multiple comparisons

$\begin{array}{lllll}\text { R middle/superior temporal gyrus } & 61 & -25 & 0 & 3.09 \\ \text { (BA 21/22) } & 66 & -27 & 1 & 3.18 \\ & 69 & -29 & 3 & 3.11\end{array}$

Coordinates and $Z$ scores for voxels in which there were significant activation foci when the visual discrimination task was compared to the baseline. $\mathrm{L}=$ left hemisphere; $\mathrm{R}=$ right hemisphere. $\mathrm{x}, y, \mathrm{z}$ (in $\mathrm{mm}$ ) refer to coordinates in the Talairach space [56].

\subsubsection{Dual task minus both single tasks (auditory and visual)}

Increases in cerebral activity $(P<0.05$ corrected for multiple comparisons $)$ when the dual-task paradigm was compared to the processing of both single discrimination tasks (contrast used: [2-1 -1 ] ; 2 for dual, -1 for single task) were found in the left inferior frontal sulcus (BA 9/46), the left anterior prefrontal cortex (BA 10 and BA 11/47), the left posterior middle frontal gyrus (BA 6), the left inferior parietal gyrus and intraparietal sulcus (BA 40), and the left cerebellum (see Fig. 2.2 and Table 5A). It should be noted that when the subject's performance (response times) was taken as covariate of non-interest, a globally similar pattern of activity was found but at a lower significance threshold (uncorrected $P<0.001$ value). ${ }^{2}$ Thus, frontal and parietal activation could not be exclusively related to task difficulty. Finally, to be sure that activation in the dual task was greater that in any single task, we looked for patterns of activity for each contrast separately [(dual-auditory) and (dual-visual)] and we inclusively masked each contrast by the other. This analysis revealed similar results, except that activity in the left anterior prefrontal cortex was now found at uncorrected $P$ value for the contrast (dual-visual).

Since one of the characteristics of executive tasks is that there is more than one cognitive way to perform them $[11,52]$, confirmatory random analysis was also conducted to determine whether cerebral areas were consistently activated by each and every subjects. Since random analyses are rather conservative, we also reported on cerebral areas significantly activated at an uncorrected $P<0.001$ value and previously found in the fixed effect analysis. Increases in cerebral activity at $P<0.05$ corrected were confirmed in the left lateral orbital gyrus (BA 11/47) and, at uncorrected $P<0.001$ value, in the left inferior frontal sulcus (BA 9) and left inferior parietal gyrus (BA 40) (see Table 5A).

Moreover, plots of activity in these regions were displayed for the different conditions (see Fig. 4). These plots indicated that cerebral activity in the frontal and parietal regions increased during the dual-task condition and not for the reference tasks. The only exception was a region of the anterior prefrontal cortex (BA 10; coordinates: $-48,50,-4$ ), which also exhibited increased activity in the baseline condition (with reference to the discrimination tasks). It must, however, be emphasized that other voxels in this area showed the expected pattern (namely, greater activity in the dual task than in the single and baseline tasks) (see Fig. 4 for a graphic representation of the activity of sub-areas in BA 10).

\footnotetext{
2 This analysis was not done with the number of errors in each condition as a confounding variable since very few errors were actually made (see Table 1).
} 


\subsubsection{Integration task minus both single tasks (auditory and visual)}

The integration task was supposed to involve some cognitive processes similar to those active in dual-task management, as well as processes specific to the integration of several pieces of information presented simultaneously. For this reason, we reported changes in cerebral activity at the uncorrected $P<0.001$ value when the area was found to be activated in the dual-task condition. However, for areas not previously identified, a value of $P<0.05$ corrected was applied. The comparison of cerebral activity in the integration and the singletask conditions demonstrated increases in cerebral activity (uncorrected $P<0.001$ value) in the left anterior prefrontal (BA 47/10) and middle frontal gyrus (BA 6) as well as in the left inferior frontal sulcus (BA 45 and 9/46). No significant change in activity was found at a corrected $P$ value (see Table 6 and Fig. 2.3). When the subjects' performance (response times) was taken as covariate, no changes in the pattern of cerebral activity were found.

Random effect analysis was performed to explore the consistency of cerebral activity across subjects. Again, an a priori hypothesis was used on the basis of the fixed effect analysis. This analysis showed increased cerebral activity in the left lateral orbitofrontal gyrus (BA 47, corrected $P$ value) and in the left inferior frontal sulcus (BA 9/46) as well as in the left posterior middle frontal gyrus (BA 6) (uncorrected $P$ values, see Table 6). Moreover, plots of activity in these regions were determined for the different conditions (Fig. 5). These plots indicate that cerebral activity in these regions is similar in the integration and dual-task conditions.

Fig. 2. (1) Brain activation observed (A) during visual discrimination compared to auditory discrimination; (B) during auditory discrimination compared to visual discrimination. Coordinates of all significant regions are given in Tables 3 and 4, respectively. Brain areas are rendered on a standard brain conforming to stereotactic space in SPM99 (uncorrected P value < 0.001). (2) Brain activation observed during dual task compared to visual and auditory discrimination tasks: (A) fixed effect; (B) random effect. Coordinates of all significant regions are given in Table 5. Brain areas are rendered on a standard brain conforming to stereotactic space in SPM 99 [(A) corrected P value <0.05; (B) uncorrected P value < 0.001]. (3) Brain activation observed during integration task compared to visual and auditory discrimination tasks: $(A)$ fixed effect; $(B)$ random effect. Coordinates of all significant regions are given in Table 6. Brain areas are rendered on a standard brain conforming to stereotactic space in SPM 99 (uncorrected P value <0.001). (4) Brain activation observed during the dual task compared to the integration task: (A) fixed effect; (B) random effect. Coordinates of all significant regions are given in Table 5. Brain areas are rendered on a standard brain conforming to stereotactic space in SPM 99 (corrected P value < 0.05).

(1)

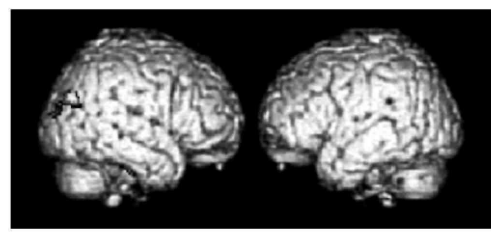

(A) Visual task - auditory task

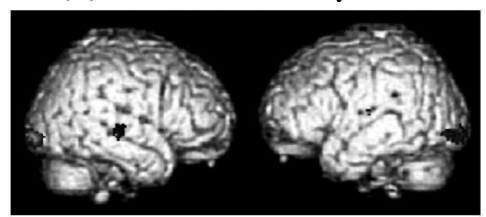

(B) Auditory task - visual task

(2)

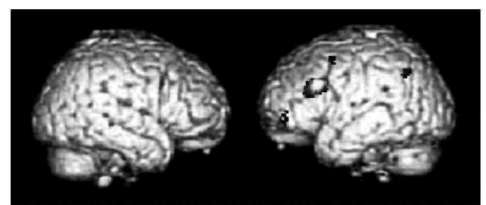

(A) Dual task - discrimination tasks (fixed effect)

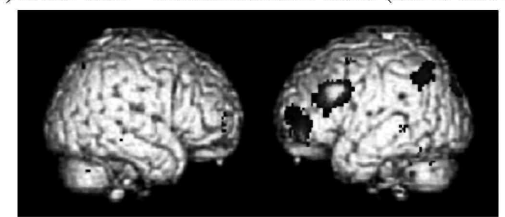

(B) Dual task - discrimination tasks (random effect)
(3)

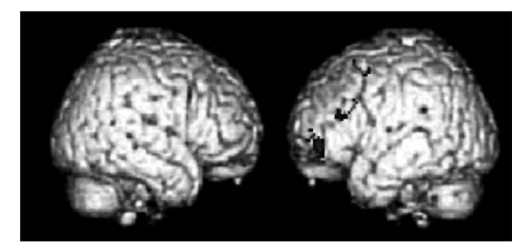

(A) Integration task - discrimination tasks (fixed effect)

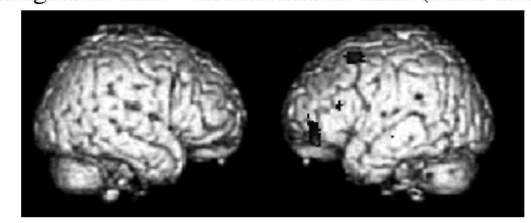

(B) Integration task - discrimination tasks (random effect)

(4)

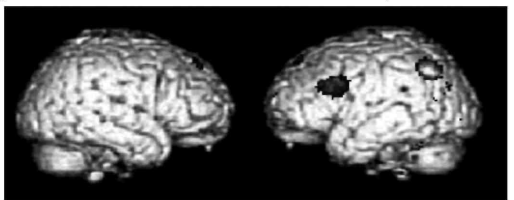

(A) Dual task - integration task (fixed effect)

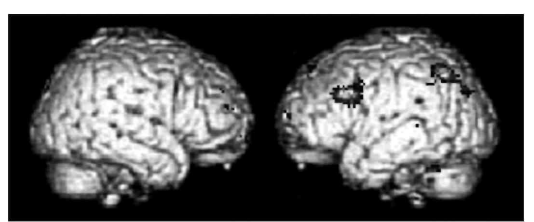

(B) Dual task - integration task (random effect) 
Table 3. Regions with significant rCBF changes in visual versus auditory discrimination task

\begin{tabular}{lcccc}
\hline Brain areas & \multicolumn{3}{c}{ Stereotactic coordinates } & Z score \\
\cline { 2 - 4 } & $x$ & $y$ & $\mathrm{Z}$ & \\
\cline { 1 - 3 } Voxel $P$ value $<0.001$, uncorrected for multiple comparisons \\
R angular gyrus (BA 39) & 42 & -72 & 28 & 3.83 \\
R middle occipital (BA 19) & 40 & -87 & 17 & 3.72 \\
R superior parietal gyrus (BA 7) & 24 & -58 & 40 & 3.61 \\
R fusiform gyrus (BA 20) & 36 & -28 & -25 & 3.82 \\
& 36 & -30 & -19 & 3.41 \\
\hline
\end{tabular}

Coordinates and $\mathrm{Z}$ scores for voxels in which there were significant activation foci when the visual discrimination task was compared to the auditory discrimination task. $\mathrm{L}=$ left hemisphere; $\mathrm{R}=$ right hemisphere. $x, y, z$ (in $\mathrm{mm}$ ) refer to coordinates in the Talairach space [56].

Table 4. Regions with significant $r C B F$ changes in auditory versus visual discrimination task

\begin{tabular}{|c|c|c|c|c|}
\hline \multirow[t]{2}{*}{ Brain areas } & \multicolumn{3}{|c|}{ Stereotactic coordinates } & \multirow[t]{2}{*}{ Z score } \\
\hline & $x$ & $y$ & $z$ & \\
\hline \multicolumn{5}{|c|}{ Voxel $P$ value $<0.001$, uncorrected for multiple comparisons } \\
\hline R superior temporal gyrus (BA 22) & 69 & -23 & -1 & 3.41 \\
\hline \multirow[t]{3}{*}{ L and $\mathrm{R}$ inferior occipital gyrus (BA 18) } & -32 & -84 & -3 & 3.53 \\
\hline & -30 & -95 & -2 & 3.61 \\
\hline & 34 & -91 & 0 & 4.37 \\
\hline
\end{tabular}

Coordinates and $\mathrm{Z}$ scores for voxels in which there were significant activation foci when the auditory discrimination task was compared to the visual discrimination task. $\mathrm{L}=$ left hemisphere; $\mathrm{R}=$ right hemisphere. $x, y, z$ (in $\mathrm{mm}$ ) refer to coordinates in the Talairach space [56].

\subsubsection{Dual task minus integration task}

This comparison demonstrated significant increases in cerebral activity (corrected $P<0.05$ value) in the left deep inferior frontal sulcus (BA 44/45) and in the left inferior parietal cortex (BA 40). Random effect analysis confirmed the activity in these two areas, but this time at the uncorrected threshold (see Table 5B and Fig. 2.4). Moreover, plots of activity in these regions were determined for the different conditions (Fig. 6), and these plots indicate that cerebral activity in these regions is greater in the dual-task condition than in the other four conditions.

\subsubsection{Integration task minus dual task}

On the basis of the studies by Prabhakaran et al. [46] and Mitchell et al. [40], we predicted that the integration task would be specifically related to the left anterior hippocampus and the right medial prefrontal cortex (BA 10). However, fixed and random effect analysis demonstrated no significant foci of activity.

\subsubsection{Cerebral areas common to the dual task and integration task}

Finally, since the individual comparison of the dual and integration tasks to the single discrimination tasks demonstrated globally similar patterns of cerebral activity, we formally determined which cerebral areas are commonly activated by the dual and integration tasks using the contrast [dual - (single visual + single auditory)] masked inclusively by [integration — (single visual + single auditory)].

This analysis demonstrated significant foci of activity $(P<0.05$, corrected $)$ in the left posterior middle frontal gyrus (BA 6; $x=-34, y=12, z=55 ; \mathrm{Z}$ score $=5.07)$, and more tentatively $(P<0.001$, uncorrected) in the right middle frontal gyrus (BA 46; $x=55, y=32, z=19$; Z score $=3.36$ ), and in the cerebellum $(x=-4, y=-75, z=$ $-15 ; \mathrm{Z}$ score $=3.80)$. 
Fig. 3. Plots of relative cerebral activity in the five cognitive tasks for the voxels significantly more activated (A) in the visual versus auditory discrimination, and $(B)$ in the auditory versus visual discrimination (fixed effect analysis, uncorrected $P$ value $<0.001)$. The coordinates of each voxel are indicated on the y axis (MNI coordinates $)$ and cognitive tasks are represented on the $x$ axis $(1=$ rest $; 2=$ visual discrimination; $3=$ auditory discrimination; 4 = dual task; 5 = integration).

(A) Visual task - auditory task
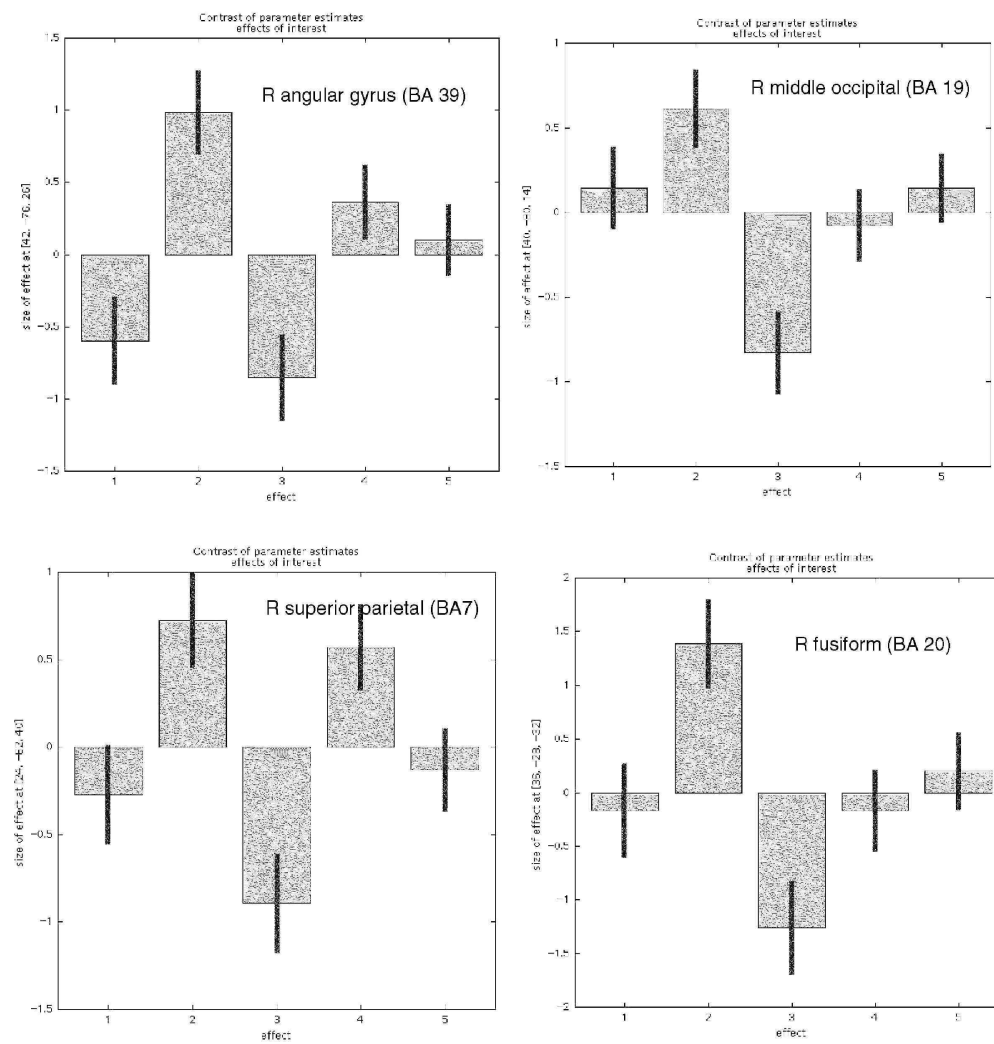

(B) Auditory task - visual task
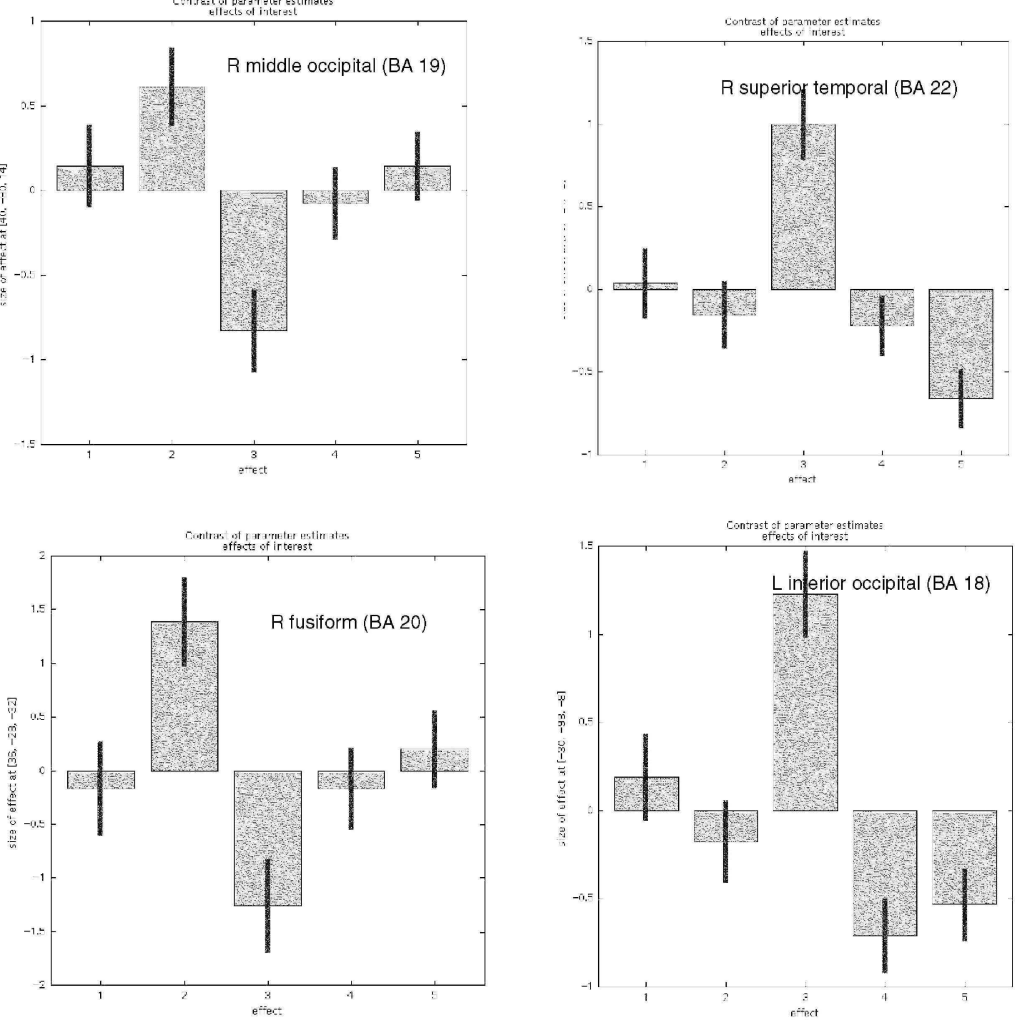

\section{DISCUSSION}

The results of this experiment can be summarized as follows. At a behavioral level, we observed a progressive increase in reaction times from the baseline to the single discrimination tasks, the integration task, and finally the dual task. From a neuroimaging perspective, the comparison of each discrimination task to the baseline did not demonstrate any activity in the prefrontal regions, although these tasks did activate posterior cortical areas (see Table 2). Secondly, in comparison to the performance of the single tasks, both dual and integration tasks were associated with increases in cerebral activity in different regions of the prefrontal cortex: inferior frontal sulcus (BA 9/46), anterior prefrontal cortex (BA 10/47), and posterior middle frontal gyrus (BA 6). In addition, the direct comparison of the dual and integration tasks demonstrated that dual-task management was associated with activation in the left inferior frontal sulcus (BA 44/45) and the left inferior parietal gyrus (BA 40) while no supplementary region was activated by the integration task. The results were not modified when behavioral performance was entered as confounding cova-riate. Finally, plots of activity demonstrated that the regions recruited during single tasks were activated to a lesser degree during the dual and integration tasks, at the exception of the right superior parietal gyrus (BA 7). 
Table 5. Regions with significant $\mathrm{rCBF}$ changes when the dual task was compared to the two single discrimination tasks and integration task

\begin{tabular}{ll}
\hline Brain areas & \multicolumn{2}{l}{ Stereotactic coordinates Z score } \\
\cline { 2 - 3 } & $x \quad \mathrm{Z}$ \\
\hline
\end{tabular}

(A) Dual task vs. discrimination tasks

Voxel $P$ value $<0.05$, corrected for multiple comparisons

$\mathrm{L}$ inferior frontal sulcus (BA 9/46)

L anterior prefrontal cortex (BA 10 and BA 11/47)

$\begin{array}{llll}-38 & 17 & 23 & 5.90 * * \\ -46 & 21 & 27 & 6.13 * \\ -51 & 15 & 27 & 5.50 * * \\ -48 & 48 & -6 & 5.15 * * * \\ -46 & 45 & 1 & 4.79 * \\ -28 & 6 & 46 & 5.34 * \\ -40 & -54 & 38 & 5.10^{* *} \\ -46 & -58 & 42 & 4.85 * \\ -6 & -84 & -18 & 5.35 *\end{array}$

L posterior middle frontal gyrus (BA 6)

L inferior parietal gyrus (BA 40)/

intraparietal sulcus

L cerebellum

$-84$

(B) Dual task vs. integration task

Voxel $P$ value $<0.05$, corrected for multiple comparisons

L inferior frontal gyrus (BA 44/45)

L inferior parietal (BA 40)

$\begin{array}{llll}-32 & 15 & 21 & 5.65^{* *} \\ -53 & -58 & 45 & 4.70^{* *}\end{array}$

Coordinates and $Z$ scores for voxels in which there were significant activation foci when the dual task was compared to the single discrimination tasks. $\mathrm{L}=$ left hemisphere; $\mathrm{R}=$ right hemisphere. $\mathrm{x}, y, \mathrm{z}$ (in $\mathrm{mm}$ ) refer to coordinates in the Talairach space [56]. *Indicates cerebral areas found to be significant for fixed effect analysis at $P<0.05$ corrected, ${ }^{*} *$ for random effect analysis at $P<0.001$ uncorrected and $* * *$ at $P<0.05$ corrected.

This study was designed to explore the neural substrates of dual-task management and the integration of information, using tasks that were not supposed to involve prefrontal activation when performed in isolation. Indeed, the comparison of each single task to the baseline actually demonstrated that these tasks do depend mainly on posterior cerebral areas. ${ }^{3}$ The auditory discrimination task was associated with increased cerebral activity in the right superior/middle temporal gyrus (BA 21/22) and the inferior occipital cortex bilaterally (BA 18), while the visual discrimination task was associated with increased cerebral activity in the right superior parietal (BA 7), angular gyrus (BA 39), occipital regions (BA 19), and right fusiform (BA 20). These cerebral areas had previously been found to be associated with sensory discrimination processes $[6,18,37,42,57]{ }^{4}$

We were interested in finding out whether dual-task management involves supplementary activity at the level of the prefrontal cortex (e.g., [20,31]) or more activity only in the posterior cerebral areas already activated by the single tasks (e.g., [10]). The comparison of dual-task management to the single tasks evidenced a left-sided fronto-parietal network, even when behavioral performance was taken as confounding covariate. Moreover, the observation of the plots of activity showed that no cerebral areas involved in the performance of single tasks exhibited similarly high activity during dual-task management, to the exception of the left superior parietal (BA 7). Taken as a whole, these results are essentially in agreement with the proposal that prefrontal areas are specifically involved in dual-task management. It must be noted that activity in the superior parietal cortex (specifically associated to the visual discrimination task) increases during dual-task coordination. The superior parietal cortex was recently associated to central executive functions such as implementation of task-related attentional sets $[16,17,58]$. At the present however, it remains unclear why such a process would be involved in the visual but not auditory discrimination task. We may also ask why cerebral areas associated with sensory

\footnotetext{
${ }^{3}$ It could be stated that discrimination tasks in fact do not engage prefrontal regions to a greater extent than baseline tasks. However, since the absence of supplementary prefrontal activity during discrimination tasks was observed using several contrasts (auditory discriminationbaseline; visual discrimination-baseline; visual-auditory discrimination, auditory-visual discrimination) and a very lenient statistical threshold, it can be properly considered that prefrontal activity is not a main characteristic of the discrimination tasks used.

4 Activity associated with auditory and visual discrimination processes was found at uncorrected $P$ values only. This low level of activity can be attributed to a strict matching of sensory stimuli between the five conditions, leading to a minimal processing of auditory stimulation in the visual discrimination task, and vice versa.
} 
discrimination tasks are less involved in dual-task management and integration tasks. Even though this study was not designed to answer this question, we can suppose that sensory processes are modulated by task difficulty (i.e., when the task becomes more difficult, fewer resources are allocated to sensory processes). In any case, the absence of increased cerebral activity in posterior regions associated with sensory processes militates against the idea that a dual task depends mainly on activity in the posterior cerebral areas (e.g., [1]).

However, unlike D'Esposito et al. [20], we did not observe activity only in the dorsolateral prefrontal cortex but in a larger antero-posterior cerebral network. Previous studies have demonstrated that the performance of various executive tasks (such as manipulation of information, inhibition, shifting, and updating) is associated with increased cerebral activity in both prefrontal and parietal areas (for a review, see [13]). These results suggest that executive functioning is based on a network of anterior and posterior cerebral areas and is not subserved by the frontal lobes alone. It is, however, unlikely that all cerebral areas highlighted in the comparison of dual task versus single tasks underlie the management of two concurrent processes.

On the contrary, various cognitive processes should intervene during dual-task management. So, since the items to process were simultaneously presented, there might be task-switching between visual and auditory processes [28]. Moreover, since no intensive training was made before scanning, strategic scheduling could also be necessary to perform the task [39]. Finally, the procedure used could also induce inhibition since the same key presses were used to respond to auditory and visual items.

By reference to the neuroimaging literature, the following roles can be proposed for these regions. Activity in the left inferior frontal sulcus, extending to the middle frontal gyrus (BA 9/46), has previously been correlated with dual-task management [20]. However, other functions have also been attributed to that region, such as manipulation of information [14,21,45], shifting processes [47], and updating of information [50,53]. More generally, Koechlin et al. [35] consider that BA 46 is engaged for selecting appropriate representations for action after the occurrence of behaviorally significant events. In our study, the activation of the left posterior part of the inferior frontal sulcus for both dual task and integration would favor a role in coordinated manipulation of different stimuli. Other frontal areas activated by dual-task management are the left posterior middle frontal and the anterior prefrontal cortex (BA 6 and BA 10/47, respectively). These areas have also frequently been associated with the functioning of the central executive of working memory. More specifically, the anterior ventrolateral activation has been reported to subserve selection in relatively complex situations, an important process in dual tasks [15]. With regard to left posterior middle frontal activation (BA 6), the role of this area has been interpreted in terms of rehearsal processes [2,44]. The left inferior parietal gyrus (BA 40) may be involved in the attentional shifting necessary to maintain simultaneously activated auditory and visual information. Indeed, several data demonstrated that parietal activation was associated to various switching tasks $[29,36]$. Moreover, this area has also been associated with attentional processes linking "external" sensory representations (in this case, the cue presented after the visual and auditory stimuli to be processed) to the relevant motor maps [17]. The posterior medial cerebellum is frequently activated for cognitive tasks, such as working-memory tasks, but its precise function remains a matter of debate. Finally, it must be noted that several paradigms exploring dual-task management have demonstrated an increase in activity in the anterior cingulate (e.g., [20,37]), attributed to response selection when two or more incompatible responses are simultaneously activated [7]. Such activity was not found in the present study but very few errors were recorded (see Table 1) and we may hypothesize that the most executive aspect of our dual-task paradigm is the simultaneous processing of the two sensory inputs.

Table 6. Regions with significant rCBF changes when the integration task was compared to the two single tasks

Brain areas

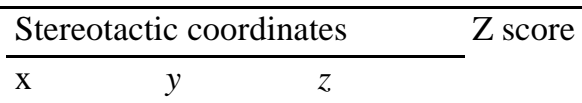

\begin{tabular}{lllll}
\hline Voxel $P$ value $<0.001$, uncorrected for multiple comparisons & & & & \\
L inferior frontal gyrus (BA 45) & -44 & 28 & 17 & $3.48^{* *}$ \\
L inferior frontal sulcus (BA 9/46) & -51 & 23 & 25 & $3.85^{*}$ \\
& -51 & 15 & 27 & $3.39 *$ \\
L posterior middle frontal gyrus (BA 6) & -34 & 12 & 55 & $4.39^{* *}$ \\
L anterior prefrontal gyrus (BA 47/10) & -51 & 42 & -11 & $3.28^{* * *}$ \\
& -50 & 44 & -4 & $3.63 * *$ \\
\hline
\end{tabular}

Coordinates and $\mathrm{Z}$ scores for voxels in which there were significant activation foci when the integration task was compared to the single discrimination tasks. $\mathrm{L}=$ left hemisphere; $\mathrm{R}=$ right hemisphere. $x, y, z$ (in $\mathrm{mm}$ ) refer to coordinates in the Talairach space [56]. * Indicates cerebral areas found to be significant for fixed effect analysis at $P<0.001$ uncorrected, **random effect analysis at $P<0.001$ uncorrected and *** at $P<0.05$ corrected. 
Fig. 4. Plots of relative cerebral activity in the five cognitive tasks for the voxels that were significantly more activated during the dual task than the visual and auditory discrimination tasks (fixed effect analysis, corrected $P$ value $<0.05)$. The coordinates of each voxel are indicated on the y axis (MNI coordinates) and cognitive tasks are represented on the $x$ axis $(1=$ rest; $2=$ visual discrimination; $3=$ auditory discrimination; $4=$ dual task; 5 = integration).
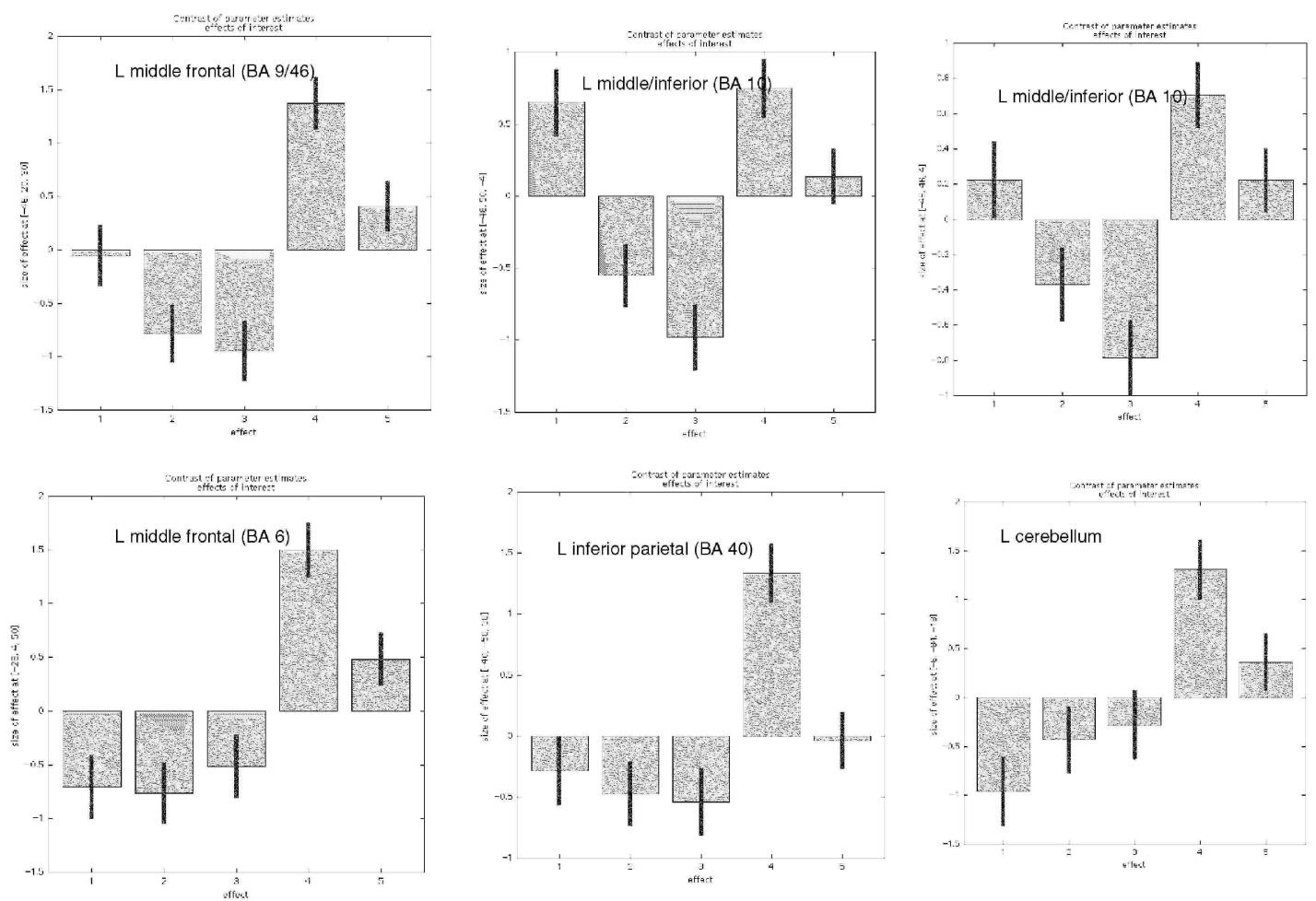

Previous fMRI studies exploring the integration of information in working memory demonstrated increased activity in the left anterior hippocampus and right medial prefrontal cortex [40,46]. No activity in these regions was found in this study. However, it must be recalled that, unlike previous studies, the integration task used was characterized by a minimal working-memory load. This could explain, at least in part, the discrepant results obtained. Moreover, only cerebral areas globally similar to those already described in the dual-task condition were found. Based on these results, it seems that the integration task we used is mainly characterized by processes similar to those involved in dual-task performance, and more specifically a coordinated manipulation of the stimuli to be matched or kept apart, respectively. However, the integration process is relatively brief and fMRI should be more appropriate for determining whether similar cerebral areas are involved in integrating sensory stimuli or in combining items stored in working memory. On the contrary, some cerebral areas are specific to the realization of the dual-task paradigm: the left inferior frontal gyrus (BA 44/45) and the left inferior parietal cortex (BA 40). According to D'Esposito et al. [21], the left inferior frontal gyrus is responsible for interference resolution. Since the same key presses were used in the dual task (but not in the integration task) to respond to auditory and visual items, activity of this region could be attributed to interference resolution during the selection of the response. As indicated above, parietal activity may be associated with the requirement to switch between the two kinds of information to be processed [29,36] or to link relevant sensory representations to motor maps [17]. Again, these two processes apply only to the dual task, since the auditory and visual information were combined in one representation in the integration task, and the response was given irrespective of the cue. 
Fig. 5. Plots of relative cerebral activity in the five cognitive tasks for the voxels that were significantly more activated during the integration task than the visual and auditory discrimination tasks (fixed effect analysis, corrected $P$ value < 0.05). The coordinates of each voxel are indicated on the y axis (MNI coordinates) and cognitive tasks are represented on the $x$ axis $(1=$ rest; $2=$ visual discrimination; $3=$ auditory discrimination; 4 = dual task; 5 = integration).
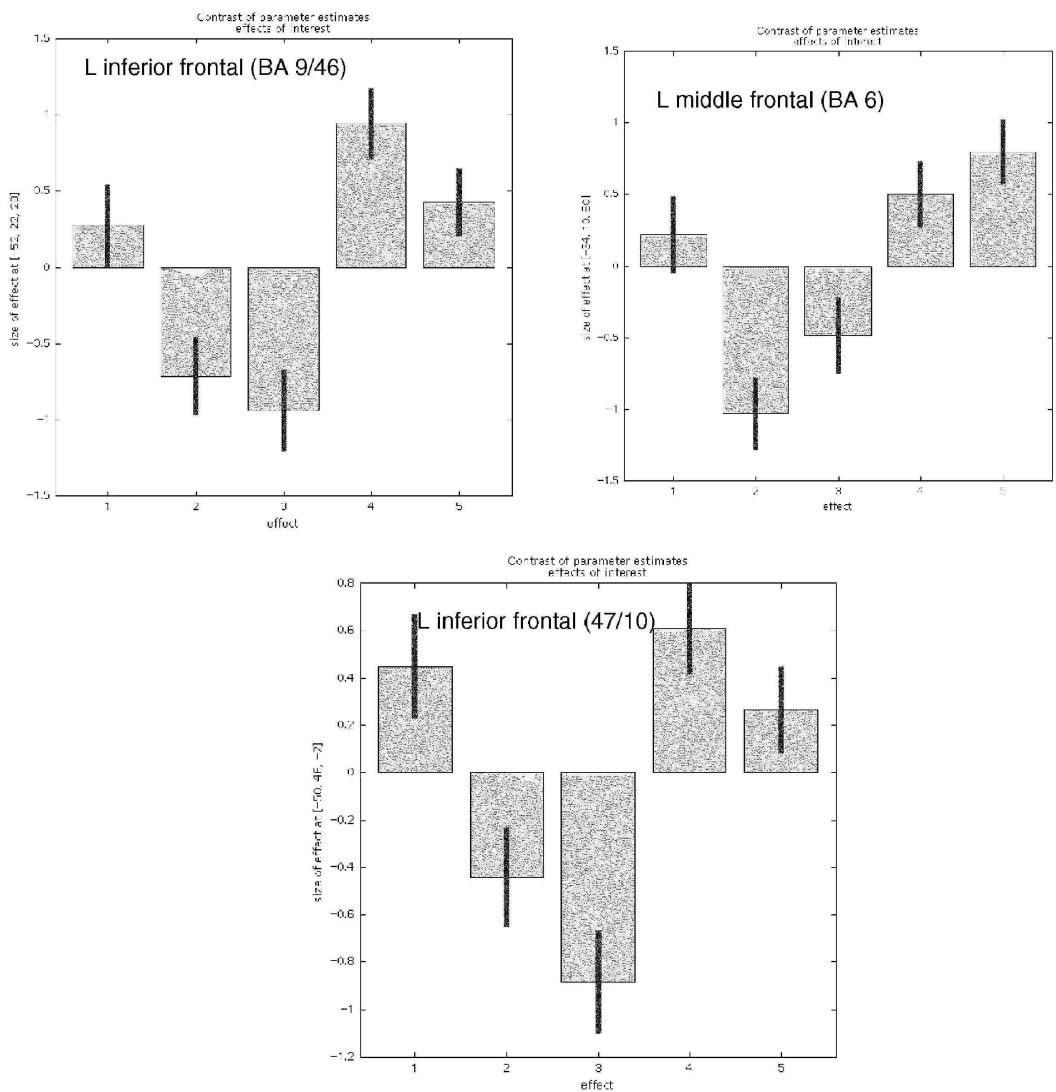

Fig. 6. Plots of relative cerebral activity in the five cognitive tasks for the voxels that were significantly more activated during the dual task than the integration task (fixed effect analysis, corrected $P$ value <0.05). The coordinates of each voxel are indicated on the y axis (MNI coordinates) and cognitive tasks are represented on the $x$ axis $(1=$ rest; $2=$ visual discrimination; $3=$ auditory discrimination; $4=$ dual task; $5=$ integration $)$.
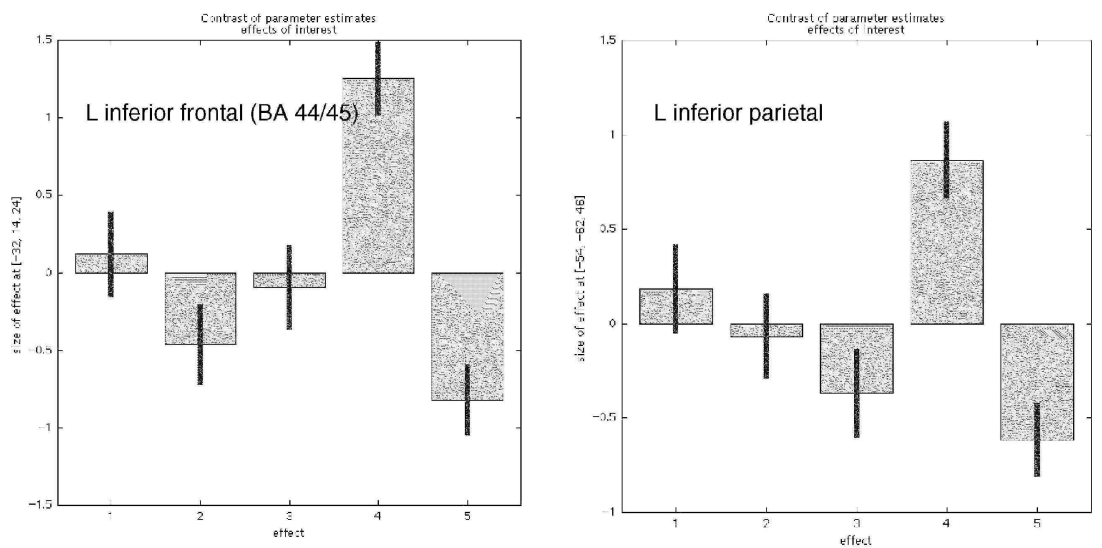
In summary, this study demonstrated that dual-task management involves specific activity of the anterior and posterior cerebral areas and that integration of two kinds of information did not require supplementary cerebral areas. However, a great number of regions were associated to dual-task coordination and the experimental design did not allow determining exactly the cognitive role underlined by these regions. Finally, an important unsolved question concerns the reason for the discrepancies between results obtained in the various studies exploring dualtask management. It seems unlikely that these discrepancies are due only to the use of control tasks that do or do not involve prefrontal areas. As suggested by Klingberg [34], another possibility might be that the overlap in the items to be processed, and consequently the shifting requirements, varied between the different dual-task paradigms. Indeed, in these paradigms, both items can be presented simultaneously or the presentation of the second item can be delayed to a greater or lesser extent (with the longest delay implying that the second item is not presented until the first one has been completely processed). In that context, Herath et al. [31] have clearly demonstrated that an increase in interference in dual-task management is associated with activity in the right inferior frontal gyrus and that this activation is spatially distinct from the cortical activity related to the main effect of dual-task performance. Moreover, Dreher and Grafman [23] demonstrated that performing two tasks in succession, as compared to simultaneously, activated a left lateral prefrontal region as well as the bilateral intraparietal sulcus. Our study was characterized by a significant overlap between visual and auditory processes, and we did in fact observe cerebral activity in an area very close to the one (coordinates: 46, 6, 26) associated by Herath et al. with increased interference in dual-task management. ${ }^{5}$ Moreover, activity in this region was also found in the integration task, which could indicate that this region is involved whenever there is an overlap between the items to be processed and not only in dual-task management.

\section{Acknowledgments}

F. Collette and S. Laureys are Research Associates at the National Fund for Scientific Research (FNRS) of Belgium. This study was supported by the Belgian National Fund for Scientific Research (FNRS), the "Fondation Médicale Reine Elisabeth" and an Interuniversity Attraction Pole (P5/04).

\section{References}

[1] R.A. Adcock, R.T. Constable, J.C. Gore, PS. Goldman-Rakic, Functional neuroanatomy of executive processes involved in dual task performance, Proc. Natl. Acad. Sci. U. S. A. 97 (2000) 3567-3572.

[2] E. Awh, J. Jonides, E.E. Smith, E.H. Schumacher, RA. Koeppe, S. Katz, Dissociation of storage and rehearsal in verbal working memory: evidence from positron emission tomography, Psychol. Sci. 7 (1996) 25-31.

[3] P. Azouvi, C. Jokic, M. Van der Linden, N. Marlier, B. Bussel, Working memory and supervisory control after closed head injury: a study of dual task performance and random generation, J. Clin. Exp. Neuropsychol. 18 (1996) 317-337.

[4] A.D. Baddeley, Exploring the central executive, Q. J. Exp. Psychol. 49A (1996) 5-28.

[5] A.D. Baddeley, S. Delia Sala, C. Papagno, H. Spinnler, Dual-task performance in dysexecutive and nondysexecutive patients with a frontal lesion, Neuropsychology 11 (1997) 187-194.

[6] R.A.B. Benedict, A.H. Lockwood, J.L. Schucard, D.W. Schucard, D. Wack, B.W. Murphy, Functional neuroimaging of attention in the auditory modality, NeuroReport 9 (1998) 121-126.

[7] M.M. Botvinick, T.S. Braver, D.M. Barch, C.S. Carter, J.D. Cohen, Conflict monitoring and cognitive control, Psychol. Rev. 108 (2001) 624-652.

[8] T.S. Braver, J.D. Cohen, L.E. Nystrom, J. Jonides, E.E. Smith, D.C. Noll, A parametric study of prefrontal cortex involvement in human working memory, NeuroImage 5 (1997) 49-62.

[9] W.H. Brouwer, R.W. Ponds, PC. Van Wolffelaar, A.H. Van Zomeren, Divided attention 5 to 10 years after severe closed head injury, Cortex 25 (1989) 219-230.

[10] S.A. Bunge, T. Klingberg, R.B. Jacobsen, J.D.E. Gabrieli, A resource model of the neural basis of executive working memory, Proc. Natl. Acad. Sci. U. S. A 97 (2000) 3573-3578.

[11] P.W. Burgess, Theory and methodology in executive function research, in: P. Rabbit (Ed.), Methodology of Frontal and Executive

\footnotetext{
${ }^{5}$ In our study, however, this activity was left-sided, which could be due to the verbal strategy used by most of the subjects for processing the items (as indicated by post hoc questioning).
} 
Published in: Cognitive Brain Research (2005), vol. 24, pp.237-251

Status: Postprint (Author's version)

Functions, Psychology Press, Hove, 1997, pp. 81-116

[12] R. Camicioli, D. Howieson, S. Lehman, J. Kaye, Talking while walking: the effect of a dual task in aging and Alzheimer's disease, Neurology 48 (1997) 955-958.

[13] F. Collette, M. Van der Linden, Brain imaging of the central executive component of working memory, Neurosci. Biobehav. Rev. 26 (2002) 105-125.

[14] F. Collette, E. Salmon, M. Van der Linden, C. Chicherio, S. Belleville, C. Degueldre, G. Delfiore, G. Franck, Regional brain activity during tasks devoted to the central executive of working memory, Cogn. Brain Res. 7 (1999) 411-417.

[15] F. Collette, M. Van der Linden, G. Delfiore, C. Degueldre, A. Luxen, E. Salmon, The functional anatomy of inhibition processes investigated with the Hayling task, NeuroImage 14 (2001) 258-267.

[16] F. Collette, M. Van der Linden, S. Laureys, G. Delfiore, C. Degueldre, A. Luxen, E. Salmon, Exploring the unity and diversity of the neural substrates of executive functioning, Hum. Brain Mapp. (in press).

[17] M. Corbetta, G.L. Shulman, Control of goal-directed and stimulus-driven attention in the brain, Nat. Rev. 31 (2002) $201-215$.

[18] M. Corbetta, F.M. Miezin, S. Dobmeyer, G.L. Shulman, S.E. Petersen, Attentional modulation of neural processing of shape, color, and velocity in humans, Science 248 (1990) 1556-1559.

[19] C.M. Cowey, S. Green, The hippocampus: a "working memory" structure? The effect of hippocampal sclerosis on working memory, Memory 4 (1996) 19-30.

[20] M. D'Esposito, J.A. Detre, CD. Alsop, R.K. Shin, S. Atlas, M. Grossman, The neural basis of the central executive of working memory, Nature 378 (1995) 279-281.

[21] M. D'Esposito, B.R. Postle, D. Ballard, J. Lease, Maintenance versus manipulation of information held in working memory: an eventrelated fMRI study, Brain Cogn. 41 (1999) 66-86.

[22] J.F. Démonet, F. Chollet, S. Ramsay, D. Cardebat, J.L. Nespoulous, R. Wise, A. Rascol, R.SJ. Frackowiak, The anatomy of phonological and semantic processing in normal subjects, Brain 115 (1992) 1753-1768.

[23] J.C. Dreher, J. Grafman, Dissociating the roles of the rostral anterior cingulate and the lateral prefrontal cortices in performing two tasks simultaneously or successively, Cereb. Cortex 13 (2003) 329-339.

[24] M.J. Emerson, A. Miyake, DA. Rettinger, Individual differences in integrating and coordinating multiple sources of information, J. Exp. Psychol. Learn. Mem. Cogn. 25 (1999) 1300-1321.

[25] KJ. Friston, CD. Frith, P.F. Liddle, R.J. Dolan, A.A. Lammertsma, R.S.J. Frackowiak, The relationship between global and local changes in PET scans, J. Cereb. Blood Flow Metab. 10 (1990) 458-466.

[26] KJ. Friston, CD. Frith, P.F. Liddle, R.SJ. Frackowiak, Comparing functional (PET) images: the assessment of significant changes, J. Cereb. Blood Flow Metab. 11 (1991) 690-699.

[27] KJ. Friston, A.P Holmes, KJ. Worsley, How many subjects constitute a study? Neurolmage 10 (1999) 1-5.

[28] H. Garavan, Serial attention within working memory, Mem. Cogn. 26 (1998) 263-276.

[29] H. Garavan, TJ. Ross, S J. Li, E.A. Stein, A parametric manipulation of central executive functioning, Cereb. Cortex 10 (2000) 585-592.

[30] J.V. Haxby, C.L. Grady, B. Horwitz, C.Ungerleider, M. Mishkin, R.E. Carson, P. Herscovitch, M.B. Schapiro, S.I. Rapoport, Dissociation of object and spatial visual processing pathways in human extrastriate cortex, Proc. Natl. Acad. Sci. U. S. A 88 (1991) 16211625.

[31] P. Herath, T. Klingberg, J. Young, K. Amunts, P. Roland, Neural correlates of dual task interference can be dissociated from those of divided attention: an fMRI study, Cereb. Cortex 11 (2001) 796-805.

[32] A. Holmes, K. Friston, Generalisability, random effects and population inference, Neurolmage 7 (1998) 754

[33] M.A. Just, P.A. Carpenter, T.A. Keller, E. Emery, H. Zajac, K.R. Thulborn, Interdependence of nonoverlapping cortical systems in dual cognitive tasks, Neurolmage 14 (2001) 417-426.

[34] T. Klingberg, Concurrent performance of two working memory tasks: potential mechanisms of interference, Cereb. Cortex 8 (1998) 593-601

[35] E. Koechlin, C. Ody, F. Kouneiher, The architecture of cognitive control in the human prefrontal cortex, Science 302 (2003) 
Published in: Cognitive Brain Research (2005), vol. 24, pp.237-251

Status: Postprint (Author's version)

1181-1185.

[36] A. Kübler, K. Murphy, J. Kaufman, E.A. Stein, H. Garavan, Coordination within and between verbal and visuospatial working memory: network modulation and anterior frontal recruitment, Neurolmage 20 (2003) 1298-1308.

[37] R. Loose, C. Kaufmann, D.P. Auer, K.W. Lange, Human prefrontal and sensory cortical activity during divided attention tasks, Hum. Brain Mapp. 18 (2003) 249-259.

[38] E.P. Lorsch, D.R. Anderson, A.D. Wells, Effects of irrelevant information on speeded classification tasks: interference is reduced by habituation, J. Exp. Psychol. Hum. Percept. Perform. 10 (1984) 850-864.

[39] D.E. Meyer, D.E. Kieras, A computational theory of executive cognitive processes and multiple-task performance: Part 1. Basic mechanisms, Psychol. Rev. 104 (1997) 3-65.

[40] K. Mitchell, M.K. Johnson, C.L. Raye, M. D'Esposito, fMRI evidence of age-related hippocampal dysfunction in feature binding in working memory, Cogn. Brain Res. 10 (2000) 189-196.

[41] P.G Nestor, R. Parasuraman, J.V. Haxby, C.L. Grady, Divided attention and metabolic brain dysfunction in mild dementia of the Alzheimer's type, Neuropsychologia 29 (1991) 379-387.

[42] D.S. O'Leary, N.C. Andreasen, R.R. Hurtig, I.J. Torres, LA. Flashman, M.L. Kesler, S.V. Arndt, T.J. Cizadlo, L.L.B. Ponto, G.L. Watkins, R.D. Hichwa, Auditory and visual attention assessed with PET, Hum. Brain Mapp. 5 (1997) 422-436.

[43] H. Pashler, Dual-task interference in simple tasks: data and theory, Psychol. Bull. 116 (1994) 220-244.

[44] E. Paulesu, CD. Frith, R.S.J. Frackowiak, The neural correlates of the verbal component of working memory, Nature 362 (1993) $342-345$.

[45] B.R. Postle, J.S. Berger, M. D'Esposito, Functional neuroanatomical double dissociation of mnemonic and executive control processes contributing to working memory performance, Proc. Natl. Acad. Sci. U. S. A. 96 (1999) 12959-12964.

[46] V. Prabhakaran, K. Narayanan, Z. Zhao, J.D.E. Gabrieli, Integration of diverse information in working memory within the frontal lobe, Nat. Neurosci. 3 (2000) 85-90.

[47] R.D. Rogers, T.C. Andrews, P.M. Grasby, D.J. Brooks, T.W. Robbins, Contrasting cortical and subcortical activations produced by attentional-set shifting and reversal learning in humans, J. Cogn. Neurosci. 12 (2000) 142-162.

[48] B. Rypma, V. Prabhakaran, J.E. Desmond, G.H. Glover, J.D.E. Gabrieli, Load-dependent roles of frontal brain regions in the maintenance of working memory, Neurolmage 9 (1999) 216-226.

[49] W. Schneider, A. Eschman, A. Zuccolotto, E-Prime user's guide, Psychology Software Tools Inc., Pittsburh, 2002.

[50] E.H. Schumacher, E. Lauber, E. Awh, J. Jonides, E.E. Smith, R.A. Koeppe, PET evidence for an amodal verbal working memory system, Neurolmage 3 (1996) 79-88.

[51] E.H. Schumacher, T.L. Seymour, J.M. Glass, D.E. Fencsik, E.J. Lauber, D.E. Kieras, D.E. Meyer, Virtually perfect time sharing in dual-task performance: uncorking the central cognitive bottleneck, Psychol. Sci. 12 (2001) 101-108.

[52] X. Seron, M. Van der Linden, P. Andrès, Le lobe frontal: à la recherche de ses spécificités fonctionnelles, in: M. Van der Linden, X. Seron, D. Le Gall, P. Andrès (Eds.), Neuropsychologie des Lobes Frontaux, Solal, Marseille, 1999, pp. 33-88.

[53] E.E. Smith, J. Jonides, R.A. Koeppe, Dissociating verbal and spatial working memory using PET, Cereb. Cortex 6 (1996) 11-20.

[54] E.E. Smith, A. Geva, J. Jonides, A. Miller, P.A. Reuter-Lorenz, R.A. Koeppe, The neural basis of task switching in working memory: effect of performance and aging, Proc. Natl. Acad. Sci. U. S. A. 98 (2001) 2095-2100.

[55] AJ. Szameitat, T. Schubert, K. Müller, D. Yves von Cramon, Localization of executive functions in dual-task performance with fMRI, J. Cogn. Neurosci. 14 (2002) 1184-1199.

[56] J. Talairach, P. Tournoux, Co-planar stereotaxic atlas of the human brain: 3-dimensional proportional system: an approach to cerebral imaging, Thieme, Stuttgart, 1988.

[57] L.G. Ungerleider, M. Mishkin, Two cortical visual systems, in: D.J. Ingle, MA. Goodale, R.J.W. Mansfield (Eds.), Analysis of Visual Behavior, MIT Press, Cambridge, MA, 1994, pp. 549-589.

[58] T.D. Wager, E.E. Smith, Neuroimaging studies of working memory: a meta-analysis, Cogn. Affect. Behav. Neurosci. 3 (2003) $255-274$

[59] K J. Worsley, A unified statistical approach for determining significant signals in images of cerebral activation, Hum. Brain Mapp. 4 
Published in: Cognitive Brain Research (2005), vol.24, pp.237-251

Status: Postprint (Author's version)

(1996) 58-73.

[60] P.L. Yee, E. Hunt, J.W. Pellegrino, Coordination cognitive information: task effects and individual differences in integrating information from several sources, Cogn. Psychol. 23 (1991) 615-680. 